\title{
Overlapping Reliable Control for a Cable-Stayed Bridge Benchmark
}

\author{
Lubomír Bakule, Fideliu Paulet-Crainiceanu, José Rodellar, and Josep M. Rossell
}

\begin{abstract}
The brief presents a reliable 1-out-2 reduced order control design strategy for a cable-stayed bridge benchmark using two overlapping subsystems and the linear quadratic Gaussian (LQG) design. Reliability with regard to controller failures is considered. Local controllers are designed for reduced order subsystems of expanded system. They are implemented and evaluated on the original overall system model. Two different sets of numerical experiments of reliable control design within 1-out-2 controller failure structure have been systematically tested using a SIMULINK scheme and compared to the benchmark sample centralized LQG design. The performance of the reliable control design has been assessed by means of given benchmark evaluation criteria. The dynamics of the closed-loop benchmark model with this multiple controller exhibits an acceptable behavior though slightly worse than in the centralized case. Thereby, the main result is a practical well working 1-out-2 reliable controller designed for a real world large-scale system. The significance of this new strategy lies in its practical systematic approach for reliable control design for large scale systems.
\end{abstract}

Index Terms-Decentralized linear quadratic Gaussian (LQG) design, inclusion principle, large scale systems, overlapping decompositions, reduced order control design, reliable control.

\section{INTRODUCTION}

A CTIVE control of flexible structures represents a new, difficult and unique problem, with many complexities in the processes of modeling, control design and implementation. Benchmark structural models have been proposed in recent years as challenging problems to the structural control community to design and compare control schemes for buildings and cable-stayed bridges subjected to seismic and wind excitations [1], [2]. The design of fault-tolerant schemes has been recently recommended as one of the important issues to be addressed for the potential implementation of control systems to real structures, in particular, control strategies able to cope with malfunction of actuators or sensors [3].

Manuscript received June 28, 2004. Manuscript received in final form October 26, 2004. Recommended by Associate Editor A. T. Vemuri. This work was supported in part by the Generalitat of Catalonia under Grant DURSI PIV, in part by the Academy of Sciences of the Czech Republic under Grant A2075304, and in part by the Committee for Science and Technology (CICYT) of Spain under Grant DPI2002-04018-C02-01.

L. Bakule is with the Institute of Information Theory and Automation, Academy of Sciences of the Czech Republic, 18208 Prague 8, Czech Republic (e-mail: bakule@utia.cas.cz).

F. Paulet-Crainiceanu is with the Department of Structural Mechanics, Faculty of Civil Engineering and Architecture "Gh. Asachi" Technical University of Iasi, 6600 Iasi, Romania (e-mail: fideliu@ @e.tuiasi.ro).

J. Rodellar is with the Department of Applied Mathematics III, Universitat Politècnica de Catalunya (UPC), 08034-Barcelona, Spain (e-mail: jose.rodellar@upc.es).

J. M. Rossell is with the Department of Applied Mathematics III, Universitat Politècnica de Catalunya (UPC), 08240-Manresa, Spain (e-mail: josep.maria.rossell@upc.es).

Digital Object Identifier 10.1109/TCST.2004.841678
On the other hand, complexity is a central problem in modern system theory and practice. The ability to study large complex systems is greatly enhanced by modern computing machinery. A theory of complex large-scale control systems is rapidly developing, supplying powerful tools that enable to solve effectively more and more practical problems in different areas [4], [5]. Due to increasing complexity of the present-day technology, reliability of control has become an essential requirement when considering the design of large systems. It has been recognized that the degree of reliability required in a high-performance design cannot be achieved merely by diligent application of standard control engineering practice, that is, by using-quality components in otherwise standard optimization techniques. For this reason, there has been a considerable effort to develop new control schemes with some form of built-in reliability enhancement. The major objective has been to synthesize a control structure so that the system performs satisfactorily under faulty conditions. Two basic failure modes can be distinguished: plant failures and controller failures. To increase the reliability regarding to controller failures, multiple control schemes have been proposed. These schemes are based on an essential redundancy achieved by multiplicity of controllers in a parallel connection, allowing for a highly reliable design using less reliable controllers. Each controller is a hot spare for the other [5], [6].

In this brief, it is attempted to explore the possibility of applying reliable multiple control tools to the cable-stayed bridge benchmark control problem proposed in [1]. This benchmark corresponds to a real-world complex 1.2-km long cable-stayed bridge which has been recently constructed in Cape Girardeau, MO. The approach proposed in this brief is based on the usage of overlapping decompositions and the inclusion principle. Reliability regarding controller failures is considered. Failure mode of a controller means that the controller is totally disconnected from the plant on a certain time interval. Intermediate situations between the normal functioning and the failure modes, where only some sensors or actuators of such controller fail, are included within the well known concept of connective stabilization. This concept is explained in detail for instance in [5, Ch. 9.3]. This means that if stability of the control system under both normal and failure modes is guaranteed, they are also guaranteed for the intermediate cases. Given the large dimensions of this bridge, a decentralized setting is adopted for the control design. Additionally to the reliability, potential advantages for designing decentralized control schemes are in the reduction of transmission costs within the feedback loop, the reduction of the overall computational effort and the possibility of using parallel implementation in real time. Although control schemes including different decompositions and decentralized control methods may offer a powerful tool for large-scale structural control system applications, their potential remains 
only partially used so far. In fact, only various decentralized control strategies have been applied in structural systems such as buildings [7]-[10], bridges [11]-[15], [10], [16], telescopes [17], [18], etc. Up to the author's knowledge, fault-tolerance issues have not been addressed in these references. Reliable control design using multiple control schemes has been addressed in [19] only.

Bridge structures offer different possibilities to define decomposition into subsystems that may be appropriate for design of reliable controllers. The benchmark problem deals with a long span cable-stayed bridge with two main towers and over hundred cables attached to. In this study, the simplest case of reliable control design using overlapping decompositions is considered. The overlapping decomposition of the bridge follows the same line as proposed in [12]. However, no reliability issues have been considered there.

Among the wide variety of methods available for reliable multiple control design, the linear quadratic Gaussian (LQG) with an infinite time horizon is adopted. The main reason for this selection is that, in order to conclude on the potential benefits of multiple control schemes, it is a standard reasonable step to compare the performance with the one of a reference centralized control scheme. In this case, we have selected the sample LQG design in [1] as a reference case.

The brief constructively describes a procedure for multiple control design using an overlapping decentralized control approach for interconnected systems in which 1-out-2 controller failure structure is included. The design starts first with the overall finite-element model (FEM) of the benchmark cable-stayed bridge decomposed into two overlapping subsystems. Each subsystem is built to include half of the bridge associated with one of the towers and their attached cables, the overlapping part being the central portion of the bridge deck where no cable is attached. Second, the original model is expanded into the model with disjoint subsystems structure using the inclusion principle [5], [7]. Then the decentralized LQG design is performed for each subsystem of the expanded model [20]. This design is made by performing a model reduction for each subsystem because of the high dimensionality of each subsystem. The effectiveness of the decentralized control approach is evaluated by means of numerical simulations. To do this, the designed local controllers are implemented into the original overall FEM model as representative of the real bridge. And, in order to measure the performance, calculation of evaluation criteria given in the benchmark problem for selected earthquake excitations is used.

To design reliable control, two sets of design sequences are considered. The first set considers both local controllers designed to satisfy the evaluation criteria when they both work together as an overlapping controller in the bridge. Then, two failure modes are tested. The first failure mode supposes only the right half part of the bridge subsystem with operating local controller. The second failure mode considers the opposite situation, i.e., only the left half part of the bridge subsystem with operating controller. In the second set of design sequences, each local controller is designed as the only controller acting on the whole bridge, that is assuming a priori the two failure modes of the first set. This means that each local control is designed for each failure mode separately, so that the evaluation criteria should be satisfied. Finally, both controllers are implemented and evaluated together as one operating overlapping control.

\section{Problem Statement}

Consider the Phase I benchmark cable-stayed bridge described in [1]. It is composed of two towers and 128 cables. The bridge is excited by an earthquake longitudinal acceleration. Five accelerometers and eight displacement sensors are used to supply feedback information for the control, which is produced by 24 hydraulic actuators located between the deck and the towers and the end supports acting to apply longitudinal forces on the deck. A complete physical description of the bridge, a finite element model and a MATLAB/SIMULINK simulation framework are given in [1] as a benchmark for control design. The model resulting from finite element formulation has the form

$$
\mathbf{M U ̈}+\mathbf{K U}=-\mathbf{M} \Gamma \ddot{x}_{g}+\mathbf{\Lambda f}
$$

where $\ddot{\mathbf{U}}$ is the second time derivative of the response vector $\mathbf{U} \in \mathbb{R}^{\mathbf{9 0 9}}$. $\mathbf{M}$ and $\mathbf{K}$ are the mass and stiffness matrices of the structure, $\mathbf{f}$ is the vector of control force inputs, $\ddot{x}_{g}$ is the longitudinal seismic ground acceleration, $\Gamma$ is a vector of zeros and ones defining the loading of the ground acceleration to the structure, and $\Lambda$ is a matrix defining how the forces produced by the control actuators enter the structure.

The objectives of this study are the following.

- Derive a systematic active control strategy for reliable controllers design using a decentralized control approach with overlapping decomposition of the benchmark bridge structure.

- Verify the proposed strategy on numerical simulations of reliable control design. Particularly, evaluate the performance of the proposed control by calculating benchmark evaluation criteria under selected benchmark earthquake excitations.

\section{SOLUTION}

This section is divided into three parts: system decomposition, reliable control design and simulation results.

\section{A. System Decomposition}

The benchmark bridge structure has two towers as main structural elements. It naturally suggests to look for a decomposition of the overall FEM model into two overlapping strongly coupled subsystems as proposed in [12] (see Fig. 1).

From a structural point of view, the tower and the deck plus the cables attached to it form a truss-like structure. This truss is very stiff compared to the connection between the two halves of the bridge, which is a small part of the deck located in the middle of the main span. This feature follows from the FEM analysis of the whole bridge. Based on this analysis, the proposed decomposition of the bridge into two parts is the most appropriate from the structural point of view. Other subdivision of the structure would lead to substructures with elements that would not be working together, in contrast with the case of a truss substructure. 

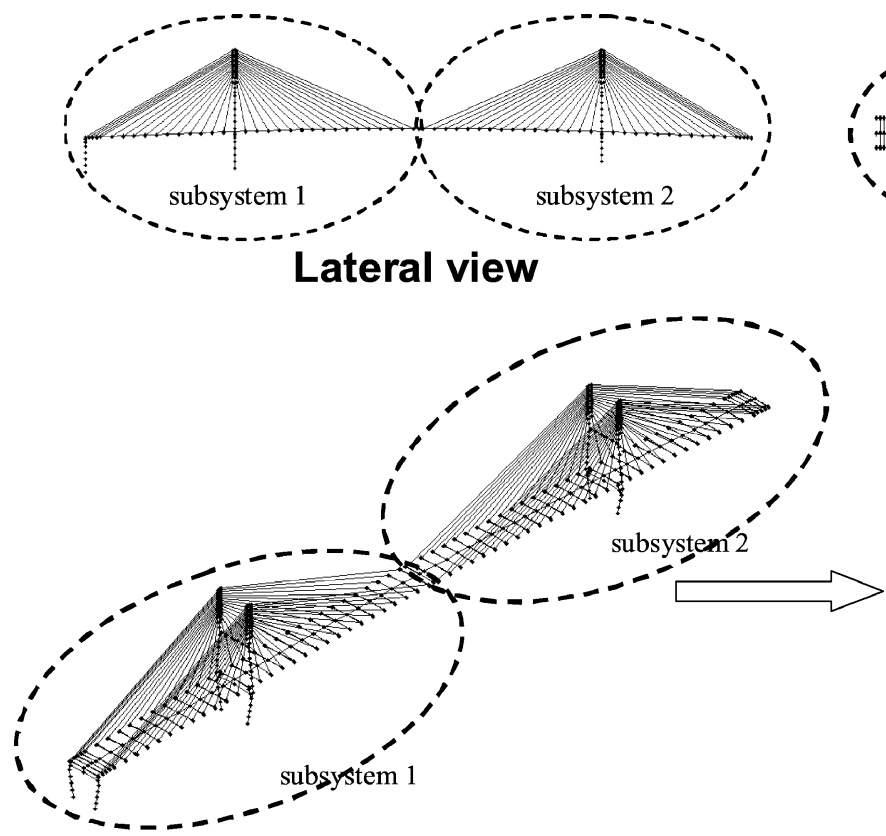

Spatial view
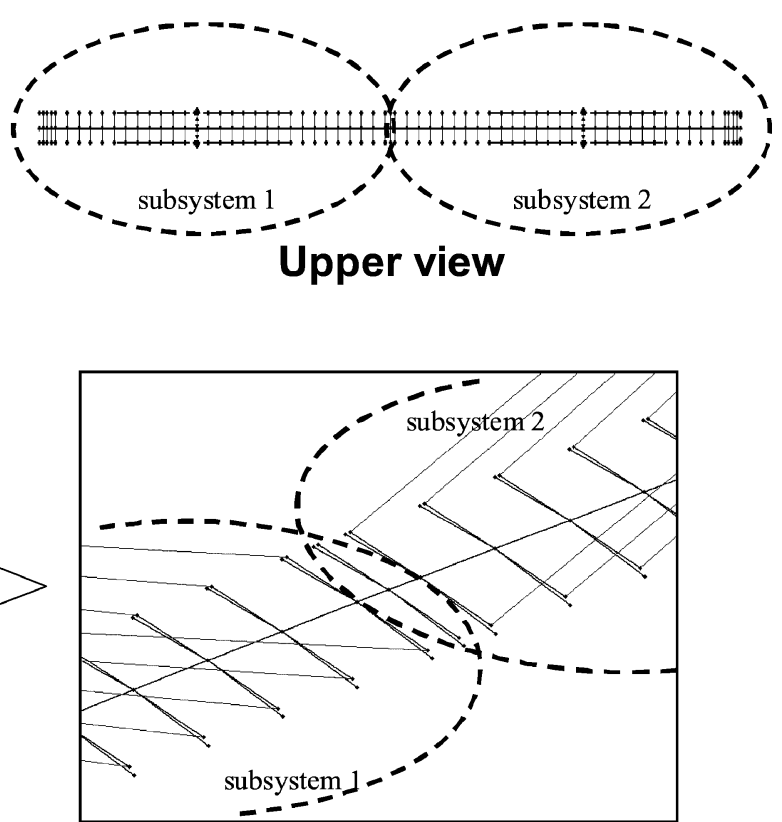

Center detailed view

Fig. 1. Bridge model and its decomposition.

Consider that the response vector $\mathbf{U}$ is appropriately rearranged into three components $\mathbf{U}=\left(\mathbf{U}_{\mathbf{1}}^{\mathbf{T}}, \mathbf{U}_{\mathbf{2}}^{\mathbf{T}}, \mathbf{U}_{\mathbf{3}}^{\mathbf{T}}\right)^{T}$. Vectors $\mathbf{U}_{1} \in \mathbb{R}^{452}$ and $\mathbf{U}_{3} \in \mathbb{R}^{452}$ include the responses of the degree-of-freedom (DOF) of the half of the bridge associated with the left and right towers and their associated cables, respectively. Vector $\mathbf{U}_{2} \in \mathbb{R}^{5}$ involves the DOF of the center of the deck where no cable is attached. Generally, there are two ways how to consider this center from the decomposition point of view. The disjoint decomposition considers this center as a weak connection between independent subsystems, while the overlapping decomposition considers this center as a strong connection which is shared by both subsystems. Because the deck is constructed as a whole, it is more appropriate to use the overlapping approach. According to these three vectors, the matrices $\mathbf{M}$ and $\mathbf{K}$ are partitioned in the form

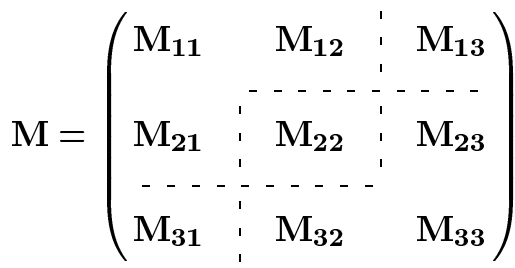

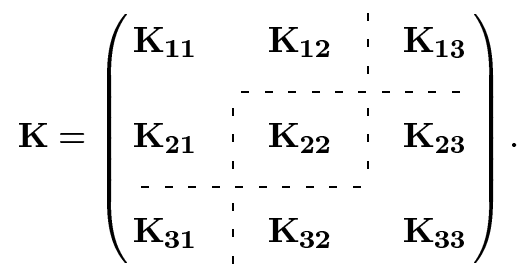

As illustrated in Fig. 1, we aim to describe the model (1) as an expanded system decomposed into two subsystems which share an overlapping part. This part corresponds to the center of the deck, that is to matrices $\mathbf{M}_{22}$ and $\mathbf{K}_{22}$. This decomposition is made by defining two vectors $\mathbf{U}_{\mathbf{1 e}}=\left(\mathbf{U}_{\mathbf{1}}^{\mathbf{T}}, \mathbf{U}_{\mathbf{2}}^{\mathbf{T}}\right)^{T}$ and $\mathbf{U}_{2 \mathrm{e}}=\left(\mathbf{U}_{2}^{\mathbf{T}}, \mathbf{U}_{3}^{\mathbf{T}}\right)^{T}$ with the common component $\mathbf{U}_{2}$. The vector $\mathbf{U}_{\mathbf{e}}=\left(\mathbf{U}_{1 \mathrm{e}}^{\mathrm{T}}, \mathbf{U}_{2 \mathrm{e}}^{\mathrm{T}}\right)^{T}$ can be related to the original vector $\mathbf{U}$ by the linear transformation $\mathbf{U}_{\mathrm{e}}=\mathbf{T U}$ with

$$
\mathbf{T}=\left(\begin{array}{ccc}
\mathbf{I}_{\mathbf{4 5 2}} & 0 & 0 \\
0 & \mathbf{I}_{\mathbf{5}} & 0 \\
0 & \mathbf{I}_{\mathbf{5}} & 0 \\
0 & 0 & \mathbf{I}_{\mathbf{4 5 2}}
\end{array}\right)
$$

where $\mathbf{I}_{(*)}$ denote the identity matrices of indicated dimensions.

A mathematical framework to establish the relations between original and expanded systems is known as the inclusion principle [5]. Essentially, it gives conditions to ensure that solutions of the expanded system (with larger dimension) include solutions of the initial system (with smaller dimension). In this work, the inclusion concept of extension is used as the concept allowing to design contractible controllers [7], that is controllers designed in the expanded system with the guarantee of being implementable in the original system. Using the transformation (3) and following the expansion process described in detail in [7], the system (1) is expanded to the following system:

$$
\mathrm{M}_{\mathrm{e}} \ddot{\mathrm{U}}_{\mathrm{e}}+\mathbf{K}_{\mathrm{e}} \mathrm{U}_{\mathrm{e}}=-\mathrm{M}_{\mathrm{e}} \Gamma_{\mathrm{e}} \ddot{x}_{g}+\Lambda_{\mathrm{e}} \mathbf{f}
$$

where the matrices $\mathbf{M}_{\mathbf{e}}$ and $\mathbf{K}_{\mathbf{e}}$ have the form

$$
\begin{aligned}
\mathbf{M}_{\mathbf{e}} & =\left(\begin{array}{llll}
\mathbf{M}_{11} & \mathbf{M}_{12} & 0 & \mathbf{M}_{13} \\
\mathbf{M}_{21} & \mathbf{M}_{22} & 0 & \mathbf{M}_{23} \\
\mathbf{M}_{21} & 0 & \mathbf{M}_{22} & \mathbf{M}_{23} \\
\mathbf{M}_{31} & 0 & \mathbf{M}_{32} & \mathbf{M}_{33}
\end{array}\right) \\
\mathbf{K}_{\mathbf{e}} & =\left(\begin{array}{cccc}
\mathbf{K}_{11} & \mathbf{K}_{12} & 0 & \mathbf{K}_{13} \\
\mathbf{K}_{21} & \mathbf{K}_{22} & 0 & \mathbf{K}_{23} \\
\mathbf{K}_{21} & 0 & \mathbf{K}_{22} & \mathbf{K}_{23} \\
\mathbf{K}_{31} & 0 & \mathbf{K}_{32} & \mathbf{K}_{33}
\end{array}\right)
\end{aligned}
$$


Note that the coupling terms in (5) are weak now, taking into account that each half of the bridge behaves like a truss-like structure as explained previously. This means that the influence of these couplings on the overall system dynamics is small. Then, by neglecting the couplings in matrices $\mathbf{M}_{\mathbf{e}}$ and $\mathbf{K}_{\mathbf{e}}$ in the left-hand side of (4), the expanded system is decoupled into two independent subsystems characterized by the matrices

$$
\begin{aligned}
\mathbf{M}_{\mathbf{d e}} & =\left(\begin{array}{cccc}
\mathbf{M}_{11} & \mathbf{M}_{12} & 0 & 0 \\
\mathbf{M}_{21} & \mathbf{M}_{22} & 0 & 0 \\
0 & 0 & \mathbf{M}_{22} & \mathbf{M}_{23} \\
0 & 0 & \mathbf{M}_{32} & \mathbf{M}_{33}
\end{array}\right) \\
\mathbf{K}_{\mathbf{d e}} & =\left(\begin{array}{cccc}
\mathbf{K}_{11} & \mathbf{K}_{12} & 0 & 0 \\
\mathbf{K}_{21} & \mathbf{K}_{22} & 0 & 0 \\
0 & 0 & \mathbf{K}_{22} & \mathbf{K}_{23} \\
0 & 0 & \mathbf{K}_{32} & \mathbf{K}_{33}
\end{array}\right) .
\end{aligned}
$$

This decoupled system is an artificial system from civil engineering point of view. However, it is convenient for model reduction and subsequent local controllers design.

\section{B. Reliable Control Design}

Suppose that a single system is controlled by several controllers operating independently. Suppose further that each controller has assigned its reliability. It is well known that different parallel structures of controller configurations may increase the overall reliability of control structures [6]. The bridge decomposition offers a natural usage of two local controllers. We consider the control structure 1-out-2. It means that we have available two parallel controllers, where anyone of them can fail and the system still will operate within an acceptable dynamic behavior. Suppose that each controller has the same reliability $p$. Then the reliability of the overall control structure denoted as $\chi(p)$ increases according to the known formula $\chi(p)=1-(1-p)^{2}$ [5].

In this brief, we suppose that the reliability design of control structure has been performed in the sense that the reliability of each controller $p$ satisfies the a priori requirement on the reliability of the overall control structure within the considered 1-out-2 structure.

Reliability issues concern the structure of interconnections of controllers. However, such structures are not related with the procedure to design the parameters of the individual controllers to satisfy the dynamic requirements on closed-loop systems. Therefore, once a reliability control structure is adopted, it is necessary to consider an appropriate scenario for the design of the controllers, including performance evaluation. Regarding the bridge, a scenario with two sets of numerical experiments has been proposed to design reliable control in a systematic way. Each set considers three consequent control design trials performance follows:

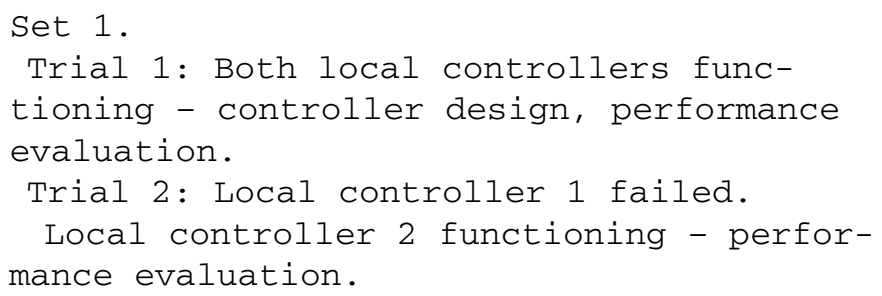

Trial 3: Local controller 1 functioning performance evaluation.

Local controller 2 failed.

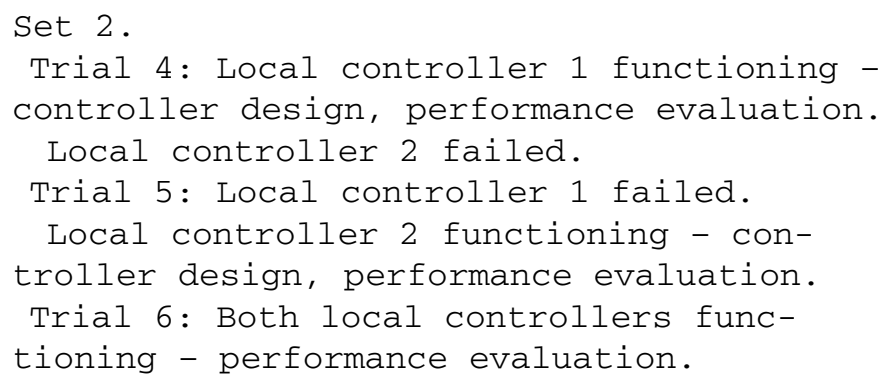

Fig. 2 gives a flowchart diagram of the steps followed in the design. Each subsystem is first subject to static condensation, inclusion of damping based on modal damping approach, and finally transformed into the state-space form [1]. The benchmark sample centralized LQG design has been selected as a [1]. The same locations and models of sensors and actuators as in the reference case have been adopted in the decentralized control design. Thus, finally, the expanded subsystems have 414 and 434 states, 4 and 4 control inputs, and 7 and 7 measured outputs, respectively.

It is clear that both subsystems are high dimensional. Therefore, model reduction must be applied first on each subsystem. Model reduction forms a balanced realization and then condenses out the states with relatively small controllability and observability grammians. The order selection criteria is to find a minimal order of the subsystems states ensuring the stability of the reduced order models. Then, a local control law is formulated for each reduced order subsystem design model by using the LQG design. This design results in the state feedback control gain matrices $\mathbf{G}_{\mathbf{1 e}}, \mathbf{G}_{\mathbf{2}}$ and the observer gain matrices $\mathbf{L}_{\mathbf{1 e}}$, $\mathbf{L}_{\mathbf{2}}$ for both subsystems. The overall decentralized control gain matrix for the expanded system is then

$$
\begin{aligned}
& \mathbf{G}_{\mathbf{e}}=\left(\begin{array}{cc}
\mathbf{G}_{1 \mathrm{e}} & 0 \\
0 & \mathbf{G}_{\mathbf{2 e}}
\end{array}\right)=\left(\begin{array}{cccc}
\mathbf{G}_{11 \mathrm{e}} & \mathbf{G}_{\mathbf{1 2 e}} & 0 & 0 \\
0 & 0 & \mathbf{G}_{\mathbf{2 3 e}} & \mathbf{G}_{\mathbf{2 4 e}}
\end{array}\right) \\
& \mathbf{L}_{\mathbf{e}}=\left(\begin{array}{cc}
\mathbf{L}_{\mathbf{1 e}} & 0 \\
0 & \mathbf{L}_{\mathbf{2 e}}
\end{array}\right)=\left(\begin{array}{cccc}
\mathbf{L}_{\mathbf{1 1 e}} & \mathbf{L}_{\mathbf{1 2 e}} & 0 & 0 \\
0 & 0 & \mathbf{L}_{\mathbf{2 3 e}} & \mathbf{L}_{\mathbf{2 4 e}}
\end{array}\right) \text {. }
\end{aligned}
$$

By using the transformation $\mathbf{T}$, this gain matrix is contracted for the implementation into the original FEM model, resulting in

$$
\begin{aligned}
\mathbf{G} & =\mathbf{G}_{\mathbf{e}} \mathbf{T}=\left(\begin{array}{ccc}
\mathbf{G}_{11 \mathrm{e}} & \mathbf{G}_{12 \mathrm{e}} & 0 \\
0 & \mathbf{G}_{23 \mathrm{e}} & \mathbf{G}_{\mathbf{2 4 e}}
\end{array}\right) \\
\mathbf{L} & =\mathbf{L}_{\mathbf{e}} \mathbf{T}=\left(\begin{array}{ccc}
\mathbf{L}_{11 \mathrm{e}} & \mathbf{L}_{12 \mathrm{e}} & 0 \\
0 & \mathbf{L}_{23 \mathrm{e}} & \mathbf{L}_{\mathbf{2 4 e}}
\end{array}\right) .
\end{aligned}
$$

The overall control is implemented by means of two local independent controllers. Local controller 1 is defined by the pair of gain matrices $\mathbf{G}_{\mathbf{1}}=\left(\mathbf{G}_{\mathbf{1 1} \mathbf{e}}, \mathbf{G}_{\mathbf{1 2 e}}\right)$ and $\mathbf{L}_{\mathbf{1}}=\left(\mathbf{L}_{\mathbf{1 1 e}}, \mathbf{L}_{\mathbf{1 2 e}}\right)$. Local controller 2 is defined by the pair of gain matrices $\mathbf{G}_{\mathbf{2}}=$ $\left(\mathbf{G}_{23 \mathrm{e}}, \mathbf{G}_{\mathbf{2 4 e}}\right)$ and $\mathbf{L}_{\mathbf{2}}=\left(\mathbf{L}_{\mathbf{2 3 e}}, \mathbf{L}_{\mathbf{2 4 e}}\right)$.

These local controllers are implemented into the original FEM system model and the dynamical behavior of the 


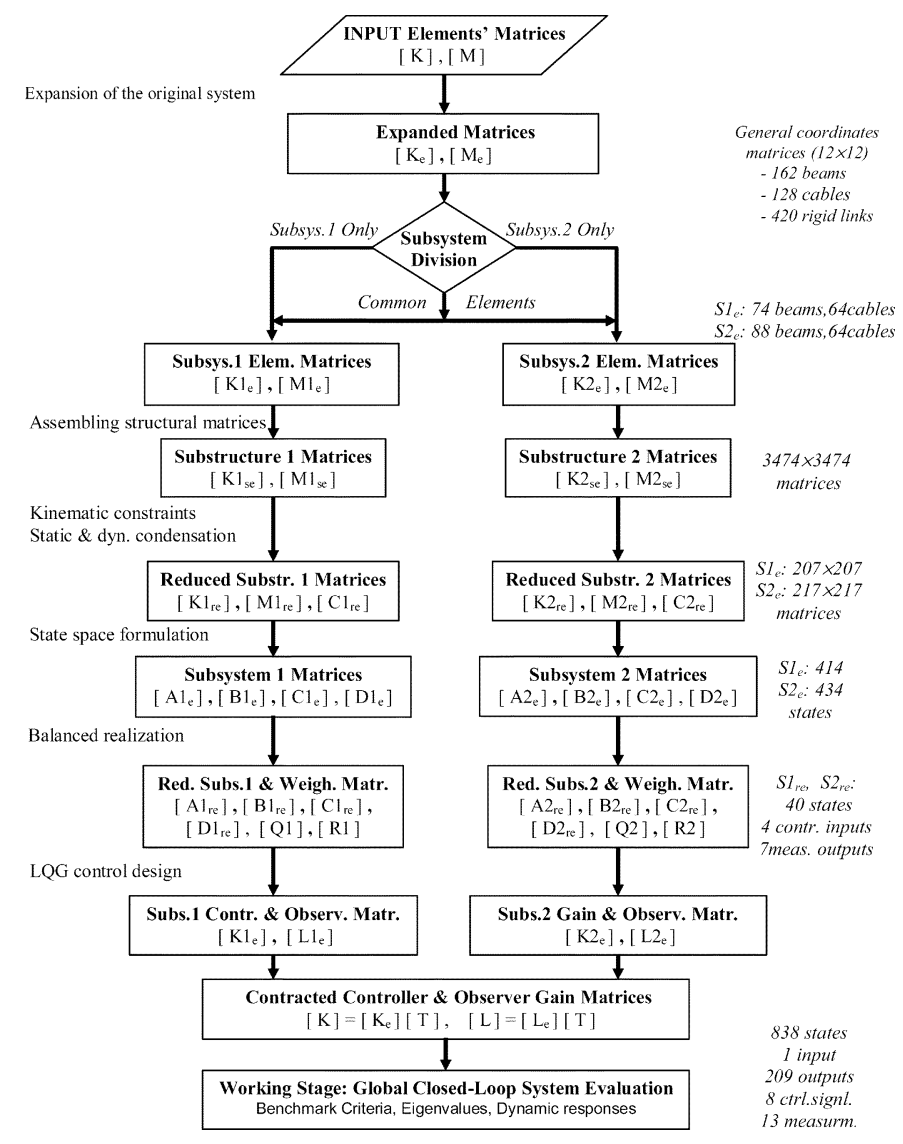

Fig. 2. Flow diagram of the design steps.

closed-loop system is tested by simulations. The results obtained by computing the given benchmark evaluation criteria are evaluated in comparison to the centralized control design reference case. The most relevant issue in this evaluation is the analysis of failure modes of the control.

\section{Simulation Results}

The model reduction and the LQG design are performed using well-known algorithms. MATLAB/SIMULINK and Control System Toolbox [21]-[23] are used to help in this design and also to perform the numerical evaluations. Fig. 3 shows the SIMULINK diagram with the two local controllers.

For the decomposition of the FEM model described previously, the model reduction results in reduced order stable subsystems with a minimal dimension of 40 states for each subsystem.

For the reliable LQG control design, a performance criterion with weights on the two displacements of the deck at the corresponding end of the bridge is selected. Also, the performance criterion involves weights on the four control signals. These weights are chosen trying to achieve uniform maximum forces for the actuators.

In order to assess the effectiveness and the potential of these reliable control schemes, two sets of numerical experiments have been performed as proposed. One set considers three different operating scenarios. In the first scenario, each decentralized controller is designed by tuning the weights in such a way that, when they are both implemented into the bridge, a
First Generation Benchmark Control Problem for Cable-Stayed Bridges Overlapping Control

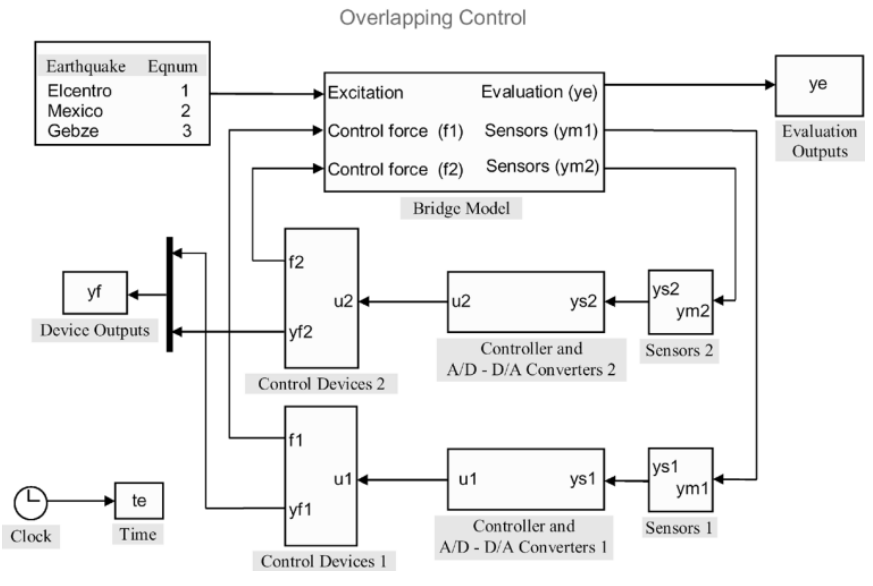

Fig. 3. SIMULINK diagram of the multiple control scheme.

satisfactory control performance is achieved and the actuator forces are kept under their saturation limit. This scenario is referred to as Trial 1. Trial 2 defines an operating scenario in which the left half of the bridge (Subsystem 1) is without control due to a failure of the corresponding decentralized controller, while the right part (Subsystem 2) remains under the controller as set for Trial 1 . Trial 3 considers the opposite situation, i.e., the subsystem 1 with control and the subsystem 2 without control.

The second set of experiments includes three more operating situations. In the first one, referred to as Trial 4, the subsystem 2 is without control but the controller of subsystem 1 is tuned trying to get satisfactory performance for the full bridge. The second operating condition (Trial 5) considers the opposite. Finally, in Trial 6, the two controllers designed in Trials 4 and 5 work together. In summary, Trials 1 and 6 correspond to normal working scenarios, while the other cases are failure (emergency) situations with only one subsystem in operation.

Figs. 4 and 5 show selected numerical results for the six trial cases. They have been performed for three external actions: El Centro (solid lines), Mexico (dashed lines with circles) and Gebze (dashed lines with x-marks) earthquakes. Fig. 4 presents the nondimensional values of more important selected performance criteria given in [1]. Criteria 1 and 3 are the peak shear force and moment at the tower bases, respectively. Criterion 6 denotes the peak deck displacement at piers. Criteria 8 and 10 are the norm values of the shear and moment at the deck level in both towers, respectively. Criterion 11 is the normed value of the deviation of the tension in the stay cables from the nominal pretension. Criterion 12 gives the maximum force generated by the control devices. Criterion 15 gives the total power required to control the bridge. The horizontal lines represent the corresponding values obtained by the benchmark given example [1], which are kept for comparison reasons.

The big number of evaluation criteria is due to the complexity of the large-scale system under control, with several structural aspects to assess which are essentially related to towers, deck, cables and actuators. Then, it is difficult and not really practical to extract very detailed conclusions from the numerical results. An overall checking of the plots in Fig. 4 tell us that, in all 

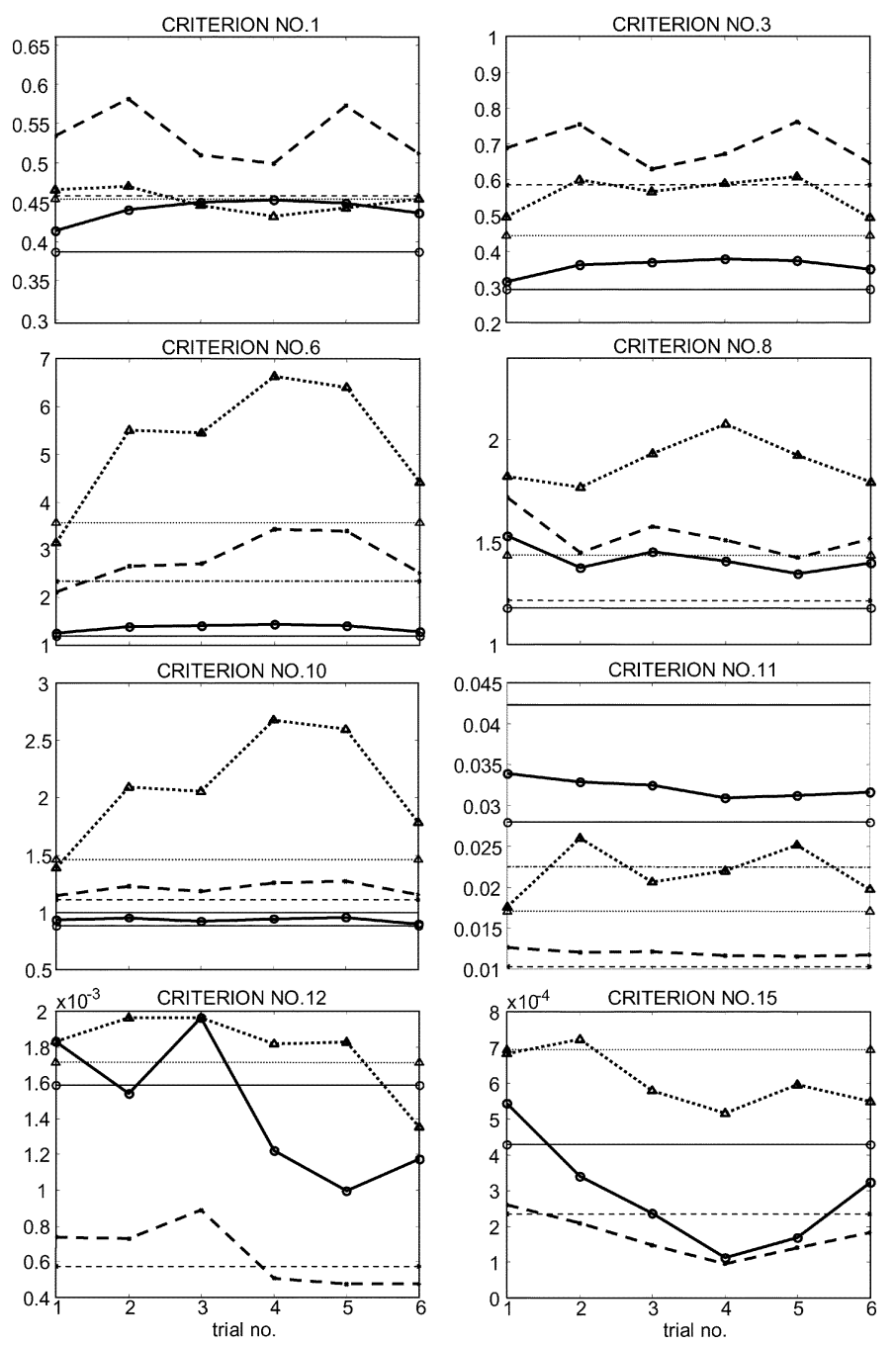

Fig. 4. Selected evaluation criteria.
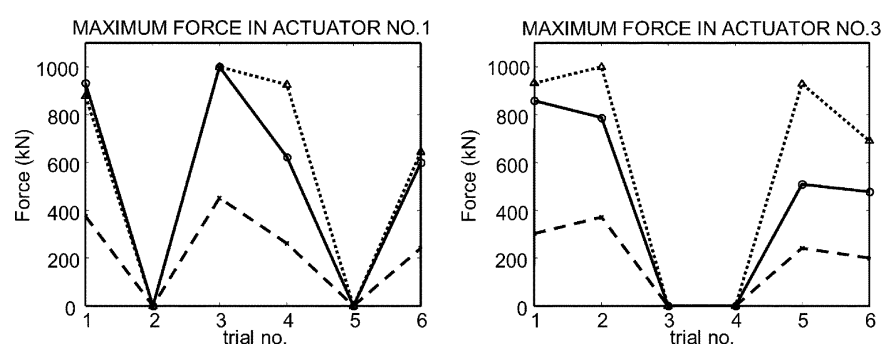

Fig. 5. Maximum actuator control forces.

the cases, the control system keeps the bridge dynamics within acceptable ranges though slightly worse than in the centralized case, which can be considered as an ideal sample reference. As expected, the Trials $2-5$, which correspond to failure modes, exhibit worse responses than the Trials 1 and 6 in which both controller are in normal operation. But, the failure responses still lie within acceptable values in terms of safety and dynamic behavior.

It is interesting to observe the Fig. 5, which gives the maximum control forces exerted by two of the actuators.

Actuator 1 belongs to local controller 1 while actuator 3 belongs to local controller 2 in Fig. 5. These plots are also very useful when tuning the values of the weighting matrices in the LQG design of the controllers. In this tuning, three main issues have been taken into account: the level of intensity of the actuator forces for each local controller, the relative level of the forces between the actuators and the balance of the forces between the controller. As expected, Trials 2 and 3 have shown that the saturation limit $(1000 \mathrm{kN})$ for the operating actuators would be reached in the case of failure of one or the other local controllers. The design starting from failures (Trials 4 and 5) leads to lower maximum forces in the actuators for the work of both subsystems (Trial 6). This fact makes the control strategy corresponding to the set 2 particularly well suited for a practical implementation. Notice that the controllers resulting from Trial 6 are different to the controllers in Trial 1. Although both sets of controllers operate well under normal conditions, saturation in the actuators appears in Trial 2 and Trial 3, which are the failure modes of the controllers of set 1 .

\section{CONCLUSION}

The brief presents a reliable 1-out-2 control design strategy for a cable-stayed bridge benchmark using two overlapping subsystems and the LQG design. Reliability with regard to controller failures is considered. Local controllers are designed for reduced order subsystems of expanded system. They are implemented and evaluated on the original overall system model. Two different sets of numerical experiments of reliable control design within 1-out-2 controller failure structure have been systematically tested using a SIMULINK scheme and compared to the benchmark sample centralized LQG design. The performance of the reliable control design has been assessed by means of given benchmark evaluation criteria. The dynamics of the closed-loop benchmark model with this multiple controller exhibits an acceptable behavior though slightly worse than in the centralized case. Thereby, the main result of the brief is a new systematic reliable control design strategy resulting in a successful design of 1-out-2 fault-tolerant reduced order controller for the large scale cable-stayed bridge benchmark by the second reliable control design strategy. From the experience with controlling the bridge, the proposed strategy appears potentially useful, in a more general sense, for systematic reliable control designs of different kinds of real world large scale systems.

\section{REFERENCES}

[1] S. J. Dyke, J. M. Caicedo, G. Turan, L. A. Bergman, and S. Hague. (2002) Phase I benchmark control problem for seismic response of cable-stayed bridges. [Online]. Available: http://wusceel.cive.wustl.edu/ quake/bridgebenchmark/pdf/J.bridge.I.4.24.02.pdf

[2] Y. Ohtori, R. E. Christenson, B. F. Spencer, and S. J. Dyke. (2001) Benchmark control problem for seismically excited nonlinear buldings. [Online]. Available: http: //www.nd.edu/ quake/papers/NLbench.pdf

[3] G. W. Housner, L. A. Bergman, T. K. Caughey, A. G. Chassiakos, R. O. Claus, S. F. Masri, R. E. Skelton, T. T. Soong, B. F. Spencer, and J. T. P. Yao, "Structural control: Past, present, and future," J. Eng. Mech. ASCE, vol. 123, no. 9, pp. 897-971, 1997.

[4] L. Bakule and J. Lunze, "Decentralized design of feedback control for large-scale systems," Kybernetika, vol. 24, no. 3-6, pp. 1-100, 1988.

[5] D. Siljak, Decentralized Control of Complex Systems. New York: Academic, 1991.

[6] K. Suyama, "Systematization of reliable control," in Proc. Amer. Control Conf., vol. 6, Piscataway, NJ, 2002, pp. 5100-5118. 
[7] L. Bakule and J. Rodellar, "Decentralized control and overlapping decomposition of mechanical systems. Part 1: System decomposition. Part 2: Decentralized stabilization," Int. J. Control, vol. 61, no. 3, pp. $559-587,1995$.

[8] L. Bakule and J. Bôhm, "Robust decentralized $\mathrm{H}^{\infty}$ control of input delayed interconnected systems," in Smart Structures. Amsterdam, The Netherlands: Kluwer, 1999, NATO Series, pp. 1-8.

[9] S. M. Kim, S. J. Elliot, and M. J. Brennan, "Decentralized control for multichannel active vibration isolation," IEEE Trans. Contr. Syst. Technol., vol. 9, no. 1, pp. 93-100, Jan. 2001.

[10] F. Paulet-Crainiceanu, "Structural active control for buildings and bridges in seismic areas," in Proc. Int. Conf. Advanced Problems Vibration Theory and Applications (ICAPV'O0), Beijing, China, 2000, pp. 147-151.

[11] L. Bakule, F. Paulet-Crainiceanu, and J. Rodellar, "Decentralized control design for a cable-stayed bridge benchmark," in Proc. Amer. Control Conf., vol. 4, Piscataway, NJ, 2002, pp. 3046-3051.

[12] L. Bakule, F. Paulet-Crainiceanu, J. Rodellar, and J. M. Rossell, "Decentralized overlapping control design for a cable-stayed bridge benchmark," in Proc. 3rd World Conf. Structural Control, vol. 2, 2003, pp. 869-874.

[13] N. Luo, J. Rodellar, M. de la Sen, and J. Vehí, "Decentralized active control of a class of uncertain cable-stayed flexible structures," Int. J. Control, vol. 75, no. 4, pp. 285-296, 2002.

[14] M. E. Magana and J. Rodellar, "Nonlinear decentralized active tendon of cable-stayed bridges," J. Struct. Control, vol. 5, pp. 45-62, 1998.
[15] F. Paulet-Crainiceanu, "Seismic response control of long cable-stayed bridges," in Proc. 2nd World Conf Structural Control, Chichester, U.K., 1999, pp. 959-964.

[16] F. Paulet-Crainiceanu, J. Rodellar, and C. Monroy, "Control settings and performance analysis of an optimal active control method for cable bridges," in Proc. 2nd Eur. Conf. Computational Mechanics (ECCM'01), Datacomp, Cracow, Poland, 2001, pp. 1-16.

[17] K. Li, E. B. Kosmatopoulos, P. Ioannou, and H. Boussalis, "Centralized, decentralized, and overlapping control designs for a segment telescope," in Proc. Int. Symp. Intelligent Control/Intelligent Systems and Semiotics, Piscataway, NJ, 1999, pp. 173-178.

[18] G. S. West-Vukovich, E. D. Davison, and P. C. Huges, "The decentralized control of large flexible space structures," IEEE Trans. Autom. Control, vol. AC-29, no. 10, pp. 866-879, Oct. 1984.

[19] L. Bakule, F. Paulet-Crainiceanu, and J. Rodellar, "Reliable control design for a cable-stayed bridge benchmark," in Proc. Amer. Control Conf., Piscataway, NJ, 2003, pp. 5040-5045.

[20] X. B. Chen and S. S. Stankovic, "Overlapping decomposition and decentralized LQG control for interconnected power systems," in Proc Int. Conf. Systems, Man, and Cybernetics, Piscataway, NJ, 1996, pp. 1904-1909.

[21] Matlab. User's Guide. Natick, MA: The MathWorks Inc., 1999.

[22] Simulink. Using Simulink. Natick, MA: The MathWorks Inc., 1999.

[23] Control System Toolbox. User's Guide. Natick, MA: The MathWorks Inc., 1999. 